\title{
Corela
}

Cognition, représentation, langage

HS-18 | 2015

La reformulation : usages et contextes

\section{La reformulation : usages et contextes}

\section{Claudine Garcia-Debanc}

\section{OpenEdition}

\section{Journals}

Édition électronique

URL : http://journals.openedition.org/corela/4032

DOI : $10.4000 /$ corela.4032

ISSN : 1638-573X

\section{Éditeur}

Cercle linguistique du Centre et de l'Ouest - CerLICO

\section{Édition imprimée}

Date de publication : 15 novembre 2015

ISSN : 1638-5748

\section{Référence électronique}

Claudine Garcia-Debanc, «La reformulation : usages et contextes », Corela [En ligne], HS-18 | 2015, mis en ligne le 15 novembre 2015, consulté le 30 avril 2019. URL : http://journals.openedition.org/ corela/4032 ; DOI : 10.4000/corela.4032

Ce document a été généré automatiquement le 30 avril 2019

\section{(c) (i) (2)(2)}

Corela - cognition, représentation, langage est mis à disposition selon les termes de la licence Creative Commons Attribution - Pas d'Utilisation Commerciale - Partage dans les Mêmes Conditions 4.0 International. 


\title{
La reformulation : usages et contextes
}

\author{
Claudine Garcia-Debanc
}

1 Reformulation, reprise, répétition, restitution, paraphrase, autant de termes qui désignent les opérations discursives qui permettent de reprendre un énoncé pour produire un nouvel énoncé qui n'est ni tout à fait le même ni tout à fait un autre. Le degré de similitude linguistique entre l'énoncé source et l'énoncé reformulateur peut-être très grand lorsqu'il s'agit de répétition ou plus faible, allant jusqu'à une très grande différence sémantique dans le cas d'une rectification. Les différents chercheurs peuvent avoir des définitions différentes de la reformulation.

2 Aux origines, dans les travaux fondateurs de Harris (1976), la paraphrase apparaît comme une propriété des langues, qui permet d'établir des relations transformationnelles entre des phrases d'une même langue. Fuchs (1982) propose une synthèse sur les différentes approches linguistiques de la paraphrase, en les situant par rapport à la tradition rhétorique et à la tradition logique.

Depuis une trentaine d'années, la reformulation a fait l'objet de nombreuses études chez les spécialistes de l'interaction, comme en atteste l'ouvrage collectif La Dame de Caluire, publié en 1987 par Pierre Bange. Cet ouvrage comporte plusieurs contributions analysant les interactions entre une locataire et un conseiller juridique, qui la conseille dans la rédaction d'une lettre à son propriétaire pour refuser une augmentation de loyer (Bange et alii, 1987). La reformulation intervient ici dans la dynamique conversationnelle d'un échange spécifique : le conseiller juridique reformule en termes techniques les constats de la locataire et les traduit en formulations écrites sous la forme d'auto-dictées. Gülich et Kotschi, De Gaulmyn et Charolles (1987) proposent et affinent une typologie de ces reformulations. Les études conversationnelles s'intéressent ensuite beaucoup aux marqueurs de reformulation dans une perspective contrastive Français-Italien (Rossari, 1993) ou par la description sémantique de différents marqueurs de reformulation comme c'est-à-dire, je veux dire, au total, ou, en anglais de marqueurs moins spécifiques comme so et well (Le Bot éd., 2008). L'approche peut également privilégier une approche énonciative où « la reformulation impose dans son mieux-dit du déjà-dit » (Le Bot, 2008). 
La reformulation intéresse aussi beaucoup les spécialistes de l'acquisition (Martinot, 1994, 2000, Bernicot et alii, 2006) qui la considèrent comme un moyen privilégié d'aider les enfants à s'approprier le lexique et la syntaxe d'une langue donnée. Ainsi Clark et Chouinard (2000) étudient l'offre langagière proposée par des adultes à de jeunes enfants de deux à quatre ans, respectivement un enfant français et un enfant américain. Elles analysent comment l'adulte corrige les formulations non conventionnelles de l'enfant, que ce soit au plan phonologique, morphologique, lexical ou syntaxique et comment l'enfant ratifie cette correction par une "acceptation tacite», "en ne réitérant pas l'erreur qui avait suscité la reformulation de l'adulte » ou par une « acceptation explicite en reprenant la reformulation en entier ou en partie", ou encore manifeste un rejet explicite (Clark, Chouinard, $2000: 18$ ). L'étude quantitative des acceptations et des rejets suite aux reformulations adultes fait apparaître un très grand nombre d'acceptations tacites, et dans une moindre mesure, d'acceptations explicites et un très petit nombre de rejets explicites. Ces résultats montrent l'attention que les enfants accordent aux reformulations des adultes et leur place centrale dans le processus d'acquisition de la langue. L'étude linguistique des reformulations permet également de disposer de traces des acquisitions en cours (Martinot, 2000).

Les spécialistes de didactique des langues recourent à des tâches de reformulation orale (Besse, 1985, Blondel, 1996) ou écrite (Charolles, Coltier, 1986 ; Kara, 2007) pour vérifier la compréhension d'un énoncé oral ou écrit et aider ainsi les apprenants à améliorer leurs compétences dans la langue cible. L'activité de reformulation permet ainsi d'explorer les ressources de la langue étrangère étudiée.

Enfin, en didactique du Français langue première, la reformulation, qu'elle soit réalisée par les élèves ou par l'enseignant, occupe une place importante dans les interactions scolaires. Les reformulations donnent des indices sur les représentations que se fait l'enseignant de l'objet à enseigner et de la discipline (Garcia-Debanc, 2006, 2007a). La compétence à reformuler peut être considérée comme une compétence professionnelle de l'enseignant (Garcia-Debanc, 2007b), dans la mesure où l'enseignant reprend les énoncés d'élèves pour faire progresser la présentation des savoirs en contrôlant la distance entre les formulations initiales et les formulations notées au tableau. Dans cette perspective, Garcia-Debanc et Volteau (2007) affinent les catégories proposées par Gülich et Kotschi (1987) et proposent une typologie des reformulations dans des interactions scolaires à l'école primaire dans diverses disciplines, en mettant en regard les fonctions des reformulations et leurs formes linguistiques.

7 Le présent numéro réunit des contributions qui analysent la place et la nature des reformulations dans l'acquisition et l'enseignement du français en Didactique du Français Langue Etrangère et en Didactique du français langue première. Elles résultent d'une journée d'études sur la reformulation, organisée par Stéphanie Volteau et Julie Rançon à l'ESPE de Poitiers. Chacune des contributions s'appuie sur l'analyse d'un corpus: reformulations d'un même récit par des enfants d'âges différents, échanges entre tuteurs et étudiants apprenant le français comme langue étrangère (étudiants de français langue étrangère ou élèves de collège), cours de sciences à l'école primaire en Français langue première, cours de collège et lycée, cours de première année à l'université. Les situations sont diverses. Les analyses se fondent sur des productions d'élèves et de longs extraits de transcriptions.

8 Claire Martinot montre le rôle central de la reformulation dans l'acquisition d'une langue par des jeunes enfants de 4 à 10 ans et propose des activités pour travailler les procédures 
de reformulation en situation scolaire. Elle s'appuie sur le principe mis à jour par Harris selon lequel "le mode d'organisation paraphrastique est déjà présent dans la langue » (Harris, 1976) pour définir la reformulation comme «tout processus de reprise d'un énoncé antérieur qui maintient, dans l'énoncé reformulé, une partie invariante à laquelle s'articule le reste de l'énoncé, partie variante par rapport à l'énoncé source » (Martinot, 1994). L'invariant peut ainsi être de nature sémantique, comme dans les définitions généralement proposées pour la reformulation, mais aussi de nature lexicale ou syntaxique, niveau d'analyse qui intéresse plus particulièrement Claire Martinot. Afin de pouvoir contrôler l'énoncé-source, elle propose des tâches de restitution de récits à des enfants de 4, 6, 8 et 10 ans, dans différentes langues (français, italien, croate, allemand, polonais, roumain, arabe yéménite). L'analyse porte plus particulièrement sur la reformulation des prédications. Une typologie des reformulations permet de mettre en évidence la complexification des reformulations des prédications du texte-source, en fonction de l'âge des élèves: elle distingue répétition (stricte ou approximative), reformulation avec modification de sens, reformulation paraphrastique. Parmi les reformulations paraphrastiques, sont distinguées les paraphrases sémantiques, formelles, définitoires et logiques. Une analyse de reformulations d'élèves en français permet de mettre en évidence "un mouvement de complexification corrélé à l'âge des enfants ». L'analyse des reformulations permet aussi, dans une perspective de recherche translinguistique, de repérer les zones de complexité, différentes selon les langues. L'article se termine par la proposition de quelques exercices permettant de travailler des notions syntaxiques difficiles à maitriser dans une langue donnée, en l'occurrence le français, notamment les constructions syntaxiques avec le choix des compléments de verbe ou les transformations qui font varier la diathèse (actif/passif). Ces exercices sont très utiles dans la perspective de la production écrite ou orale de discours.

Trois contributions s'intéressent aux explications lexicales en français langue étrangère. Pascale Trevisiol-okamura et Martine Marquillo-Larruy analysent la variété de moyens utilisés par des tuteurs francophones pour faire comprendre le sens de mots inconnus à des étudiants étrangers dans le cadre de visioconférences. Après avoir rappelé l'importance $d u$ concept de reformulation en didactique du français langue étrangère comme «traduction intralinguale» dans les travaux pionniers (Besse, 1995), elles analysent le caractère multimodal des explications lexicales dans un dispositif d'enseignement à distance combinant visioconférence et échanges écrits. Face à une incompréhension de l'étudiant étranger, les tuteurs recourent en effet au clavardage écrit, aux explications orales mais aussi aux gestes. Les contraintes du médium conduisent à privilégier les mimiques et les mouvements du visage et des mains. La transcription de ces échanges met en regard systématiquement les énoncés verbaux et les moyens non verbaux, qui occupent une place très importante dans ces explications. $\mathrm{Ce}$ dispositif expérimental, dans lequel les étudiants-futurs enseignants de FLE sont en situation d'explication à des étudiants étrangers et également en situation de coopérer pour mener à bien ces explications s'avère très efficace pour la formation professionnelle des futurs enseignants de FLE.

10 Julie Rançon analyse la place de la reformulation dans le traitement des difficultés lexicales d'une part en classe de français langue étrangère, d'autre part en classe de français langue première, en s'attachant plus particulièrement à des modalités d'explication d'un mot inconnu, selon qu'il désigne un objet du monde ou qu'il est abstrait. Pour ce faire, elle conçoit ce qu'on peut appeler une ingénierie didactique : trois 
mêmes extraits d'un roman de la fin du XIX' siècle, Bel Ami de Maupassant, ont été proposés à des professeurs de Français Langue Etrangère dans des universités françaises, travaillant avec des étudiants étrangers en cours d'intégration pour leurs études en France ( 2 enseignants) et à des professeurs travaillant en collège avec des classes de troisième ( 2 enseignants) ou en lycée avec des élèves de seconde ( 3 enseignants). Les interactions en classe ont été filmées et intégralement transcrites. L'analyse fait apparaitre une différence de modalités d'explication selon le caractère plus ou moins abstrait du mot à expliquer, conformément aux hypothèses initiales, l'importance des gestes, tout particulièrement en FLE et la grand nombre de marqueurs de modalisation dans les explications de termes abstraits. Le chercheur constate une très grande analogie entre les modalités d'explication en Français langue étrangère et en Français langue première.

11 Evelyne Berger analyse les reprises correctives dans les échanges à l'intérieur de groupes d'adolescents de 13 ans de Suisse alémanique apprenant le Français comme langue étrangère, confrontés à une tâche d'explication d'itinéraire, pour en évaluer le potentiel acquisitionnel. Le cadre d'analyse est constitué par les travaux en ethnométhodologie de la conversation et prend appui sur la notion de séquence potentiellement acquisitionnelle, notion proposée en didactique des langues par De Pietro, Matthey et Py (1989) à propos d'interactions exolingues entre un natif et un apprenant. L'analyse met en évidence l'importance des éléments prosodiques, notamment le débit, et d'éléments non verbaux, tout particulièrement le regard. L'article conclut à l'efficacité de reprises correctives intégrées entre pairs pour l'acquisition d'une langue seconde.

12 L'article proposé par Marie-France Roquelaure et Claudine Garcia-Debanc analyse la place et la nature d'un type particulier de reformulation, les exemples, dans des cours de Sciences du langage en première année à l'université. L'étude porte sur les enregistrements vidéo de quatre enseignants exposant aux étudiants un même contenu de connaissances, deux enseignants débutants et deux enseignants expérimentés, deux d'entre eux utilisant un diaporama et deux le tableau noir et le polycopié. Les quatre enseignants utilisent un même cours matrice, proposé également à distance. L'un des enseignants observés est le concepteur de ce cours. Les exemples sont présents en grand nombre dans les quatre enregistrements. Ils visent à faire comprendre la terminologie linguistique, en l'occurrence les différentes fonctions du langage dans le schéma de Jakobson. Ces exemples peuvent être présentés à l'écrit, dans un diaporama, et glosés à l'oral ou être formulés uniquement à l'oral. L'article propose une typologie de ces exemples et compare la place relative de ces différentes catégories d'exemples dans le discours des enseignants. Est ensuite analysé le traitement de ces exemples dans les prises de notes de quelques étudiants. Il apparaît que les exemples référant à une situation personnelle de l'étudiant, utilisés en grand nombre par les enseignants expérimentés, sont rarement notés par les étudiants. En revanche, ceux-ci notent scrupuleusement les définitions et les exemples de type "exemplaire de la classe » présents dans les diapositives.

13 Stéphanie Volteau inscrit sa contribution dans le champ de la didactique du Français Langue première. En s'appuyant sur les catégories proposées par Gülich et Kotchsi (1987), elle analyse les interactions orales dans une classe de CM2 au cours d'un projet d'enseignement scientifique portant sur la notion d'écosystème. L'analyse des reformulations vise à analyser le processus de construction des savoirs et à préciser le rôle de l'étayage de l'enseignant dans ce processus. La catégorie de reformulation la plus 
représentée est la répétition. Les répétitions, très nombreuses, sont le plus souvent initiées par l'enseignante pour valider une réponse d'élève, accompagner l'inscription au tableau d'un terme technique ou encore aider les élèves à surmonter une difficulté de compréhension d'une consigne. Ces répétitions peuvent être fidèles ou comporter une rectification phonologique ou syntaxique formulée par l'enseignant. L'analyse porte ensuite sur les explications définitoires, cruciales pour l'enseignement et l'acquisition de la terminologie scientifique, ici la différence entre zooplancton et phytoplancton. Les notions sont définies par ajustements successifs dans l'interaction, l'enseignant assurant un rôle d'étayage et institutionnalisant les acquis par l'inscription au tableau de ces termes techniques difficiles à orthographier du fait de leur construction morphologique et de leur étymologie. L'analyse de plusieurs extraits des interactions permet enfin de montrer que les reformulations ne doivent pas être exclusivement analysées dans l'enchainement local de tours de parole successifs mais que doivent être également prises en compte des reformulations différées. Celles-ci peuvent témoigner de l'intégration par les élèves des savoirs scientifiques en jeu dans l'interaction.

14 Les diverses contributions présentent un certain nombre de traits communs. Elles proposent une description linguistique précise des formes linguistiques des reformulations dans des interactions visant à la construction d'apprentissages, que ce soit en sciences, en langue première ou en Français langue étrangère. Les éléments non verbaux (regard, gestes) font également l'objet d'une analyse, dans la mesure où ils jouent un rôle important dans les explications, notamment en Français Langue Etrangère. Les différentes contributions montrent à quel point la reformulation occupe un rôle central dans la compétence d'interaction.

De plus, l'analyse des reformulations permet, en didactique des disciplines, de repérer les traces de l'intégration des savoirs, qu'ils portent sur des notions scientifiques ou sur des structures linguistiques. Certaines des contributions proposées ici s'intéressent principalement aux acquisitions linguistiques des élèves et des étudiants (articles de Claire Martinot, Pascale Trevisiol-Okamura et Martine Marquillo-Larruy et d'Evelyne Berger). D'autres travaux portent sur le discours de l'enseignant dans ses conduites discursives expositives (article de Marie-France Roquelaure et Claudine Garcia-Debanc), explicatives (article de Julie Rançon) ou dans son étayage par rapport aux formulations des élèves (article de Stéphanie Volteau). L'expertise professionnelle de l'enseignant s'évalue à sa capacité à produire des reformulations et à en susciter chez les apprenants. L'analyse des productions des étudiants permet aussi de mesurer les effets d'un dispositif innovant, tel que, par exemple, le dispositif multimodal d'échanges entre professeurs de Français langue étrangère en formation initiale et étudiants étrangers présenté dans la contribution de Pascale Trevisiol-okamura et Martine Marquillo-Larruy.

16 A travers la diversité des approches, les analyses produites dans ces travaux de recherche mettent en évidence l'importance d'un cadre d'analyse linguistique pour rendre compte des interactions en situation d'enseignement et le rôle central qu'occupe la reformulation, tout particulièrement dans une visée explicative. Ces observations et ces analyses sont utiles pour la formation initiale ou continue des enseignants du premier degré, des enseignants de français langue étrangère mais aussi des enseignants de langue du second degré et des enseignants du supérieur. 


\section{BIBLIOGRAPHIE}

BANGE, P. Ed. (1987) La dame de Caluire. Berne, Peter Lang.

BERNICOT, J., HUDELOT, C., \& SALAZAR-ORVIG, A. Eds (2006). La reprise et ses fonctions, La Linguistique, 42(2).

BESSE, H. (1985), Méthodes et pratiques des manuels de langue, Paris, Didier, Collection Essais.

BLONDEL, E. (1996) «La reformulation paraphrastique. Une activité discursive privilégiée en classe de langue », Carnets du CEDISCOR 4, en ligne URL : http://cediscor.revues.org/372.

CHAROLLES, M., COLTIER D. (1986), « Le contrôle de la compréhension dans une activité rédactionnelle : éléments pour l'analyse des reformulations paraphrastiques », Pratiques 49, Les activités rédactionnelles, Metz, p. 51-66.

CHAROLLES, M., (1987), « Spécialisation des marqueurs et spécificité des opérations de reformulation, de dénomination et de rectification » in Bange P. Ed : La dame de Caluire. Berne, Peter Lang, p. 99-122.

CLARK, E.V \& CHOUINARD, M.-M. (2000), « Enoncés enfantins et reformulations adultes dans l'acquisition du langage », Langages 140, p. 9-23.

DE GAULMYN, M., (1987) : « Actes de reformulation et processus de reformulation » dans Bange, P. (ed), La dame de Caluire. Berne, Peter Lang, p. 83-98.

DE PIETRO, J.-F., MATTHEY TIÈCHE, M. \& PY, B. (1989). « Acquisition et contrat didactique : les séquences potentiellement acquisitionnelles dans la conversation exolingue ». Dans Actes du troisième colloque régional de linguistique, Strasbourg, Université des Sciences humaines/Université Louis Pasteur, 28-29 avril 1988, 99-124.

FUCHS, C., (1982). La Paraphrase, Paris, PUF.

HARRIS, Z. (1976). Notes du cours de syntaxe, Paris, Le Seuil.

GARCIA-DEBANC, C. (2006). « Une méthodologie pour déterminer les objets effectivement enseignés : l'étude des reformulations dans l'interaction didactique. Etude de cas d'une séance conduite par un enseignant débutant en fin d'école primaire » dans Schneuwly B., ThevenazChristen T. (coord) Analyses des objets enseignés. Le cas du français, Bruxelles, De Boeck, p. 111-141.

GARCIA-DEBANC, C. (2007a), « Les modèles disciplinaires en acte dans les pratiques effectives d'enseignants débutants ", dans Falardeau E., Fisher C., Simard C., Sorin N., La didactique du français, Les voies actuelles de la recherche, Presses Universitaires de Laval, Québec, p. 43-61.

GARCIA-DEBANC, C. (2007b), « La reformulation orale : un élément de l'expertise professionnelle ", dans Talbot L., Bru M. : Des compétences pour enseigner, Presses Universitaires de Rennes, p. 151-168.

GARCIA-DEBANC C., VOLTEAU S., (2007). « Formes linguistiques et fonctions des reformulations dans les interactions scolaires ", Recherches linguistiques 29, Usages et analyses de la reformulation, Université de Metz, p. 309-340.

GULICH, E., KOTSCHI, T., (1987) : « Les actes de reformulation dans la consultation La dame de Caluire » dans Bange P. Ed : La dame de Caluire. Berne, Peter Lang, p. 15-81. 
HARRIS Z.S (1976). Notes du cours de syntaxe, Paris, Le Seuil.

KARA (éd.) (2007). Usages et analyses de la reformulation, Metz, Recherches linguistiques 29.

LE BOT M.-L. (2008). La reformulation. Marqueurs linguistiques, stratégies énonciatives ? Presses Universitaires de Rennes.

MARTINOT, C. (1994). La reformulation dans des productions orales de définitions et explications. (Enfants de maternelle), Thèse de Doctorat, Université Paris 8.

MARTINOT C. Ed (2000). Acquisition et reformulation, Langages 140, Larousse.

ROSSARI, C. (1993). Les opérations de reformulation. Analyse du processus et des marques dans une perspective contrastive français-italien, Berne, Peter Lang.

\section{AUTEUR}

\section{CLAUDINE GARCIA-DEBANC}

CLLE, UMR 5263, CNRS \& Université Toulouse 2-Jean Jaurès SFR AEF ESPE Midi-Pyrénées, Ecole interne UT2J 